\title{
BUNCH TRACING BY BUNCH BY BUNCH MEASUREMENT SYSTEM IN HLS*
}

\author{
Kai Zheng\#", J. H. Wang, J. H. Liu, Z. P. Liu, W.M. Li, B. G. Sun, Y. L. Yang \\ NSRL, University of Science and Technology of China, Hefei, Anhui 230029, P. R. China
}

\section{Abstract}

In this paper, we introduce a bunch tracing system which is based on a bunch-by-bunch $(\mathrm{BxB})$ measurement system in Hefei Light Source (HLS), and present the analysis of the experiment results. Using an in-phase gate signal and a double balance mixer to control an external trigger of $\mathrm{ADC}$, we test the reliability of the BxB system. By this system, we can trace all marked bunches in a set time slot or in manual burst mode. We can record all bunches' data during the injection, ramping, wiggler excitation and normal operation, and provide a powerful facility for machine study.

\section{INTRODUCTION}

The National Synchrotron Radiation Laboratory (NSRL) electron storage ring, with a circumference of 66 meters, operates with 45 bunches in $204.035 \mathrm{MHz}$ RF (Radio Frequency), and contains 31 BPMs (Beam Position Monitor). Each BPM has a four-button type pickups mounted in a skew of 450 . A $200 \mathrm{MeV}$ injection beam from linac and transport line ramps to $800 \mathrm{MeV}$ and operates at $200-300 \mathrm{~mA}$ in the HLS storage ring. A multicycle multi-turn injection system is used for current accumulation. A wiggler is available in the HLS.

The $\mathrm{BxB}$ measurement system is dedicated to observe the beam instability and works as a part of the transverse $\mathrm{BxB}$ feedback system in HLS[1]. Fig. 1 shows an overview of the BxB transverse feedback system of HLS, the shadow part is for one BPM detection system.

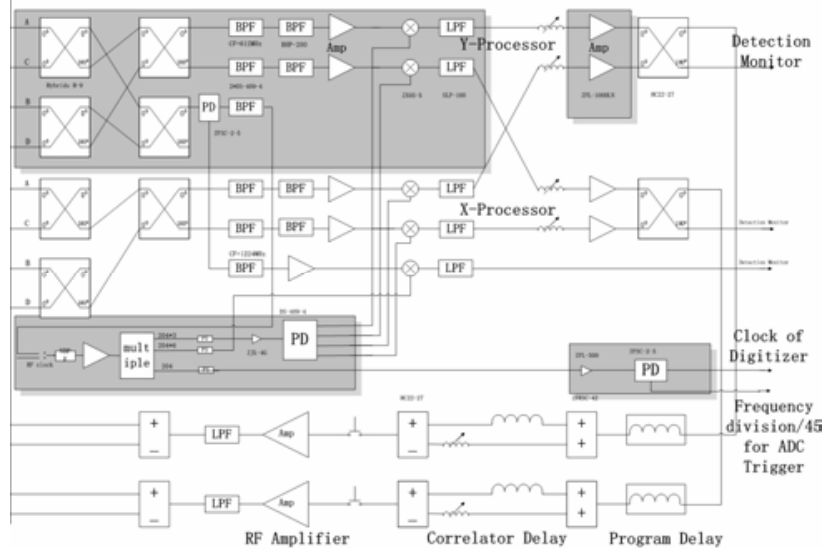

Figure 1: An overview of the transverse feedback system.

The HLS BxB transverse measurement system works at $612 \mathrm{MHz}(3 * \mathrm{fRF})$, with $100 \mathrm{MHz}$ bandwidth. A high speed 12-bit digitizer, up to 400 MSPS (Million Samples
Per Second) and simultaneous in two channels [2], is installed in a PXI (PCI eXtensions for Instrumentation) desktop. An in-phase FRF signal is used as the external clock of the ADC (Analog-to-Digital Converter), and an in-phase Revolution Frequency signal $(4.533 \mathrm{MHz})$ is connected with the External Trigger of the ADC.

\section{RELIABILITY TEST OF THE BXB MEASUREMENT SYSTEM}

Before the measurement of the beam signal, a signal simulation experiment was designed to test the reliability of the system. An overview of the test system is shown in Fig. 2.

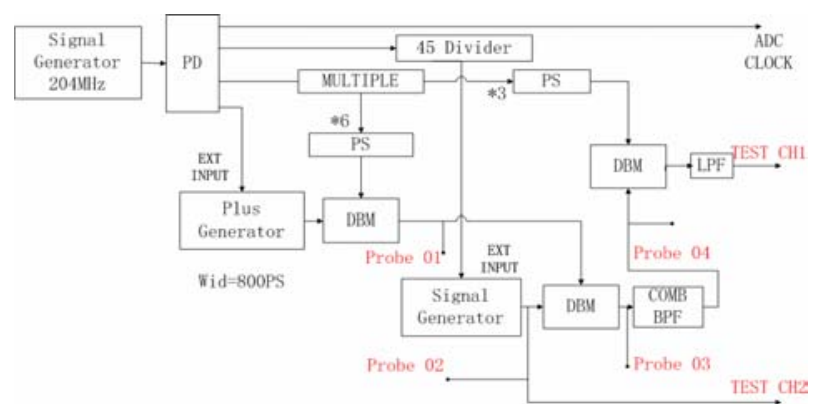

Figure 2: An overview of the reliability test system.

Controlled by a gate signal about 800 ps width, a $6 *$ fRF signal becomes a pulse signal after passing the double balance mixer (Fig. 3). This signal works as a carrier wave of the Amplitude Modulation (AM), and an inphase triangle or sine wave (Fig. 4) works as a modulation signal of the AM. The modulated pulse is treated as the beam signal, (Fig. 3). The beam signal after the comb filter is shown in (Fig. 4).

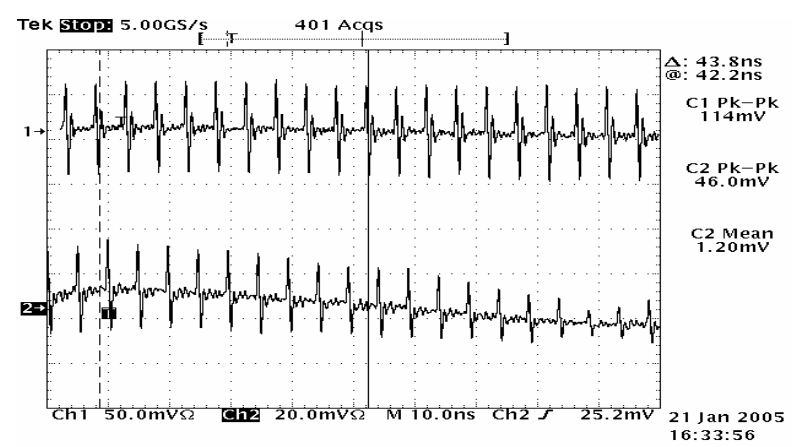

Figure 3: Pulse signal and modulated pulse signal.

*Supported by National Natural Science Project (10175063).

"kzheng@mail.ustc.edu.cn 


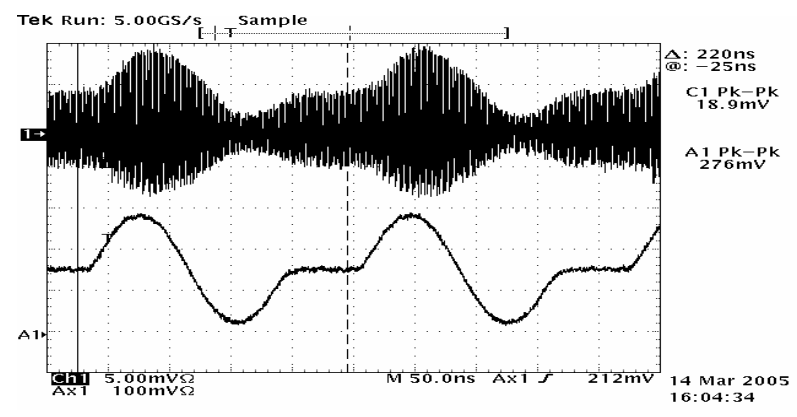

Figure 4: Modulation signal and simulation beam signal.

Figure 5 compares the original modulation signal and the modulated pulse signal processed by the $\mathrm{BxB}$ measurement system. These waveforms are detected by ADC, with 4.2M samples. The average of the Mean Square Error (MSE) (see Eq. 1) of the 45 simulation bunches' normalization amplitude is $1.49 \%$.

$$
M S E=\frac{1}{n} \sum_{i=0}^{n-1}\left(x_{i}-y_{i}\right)^{2}
$$

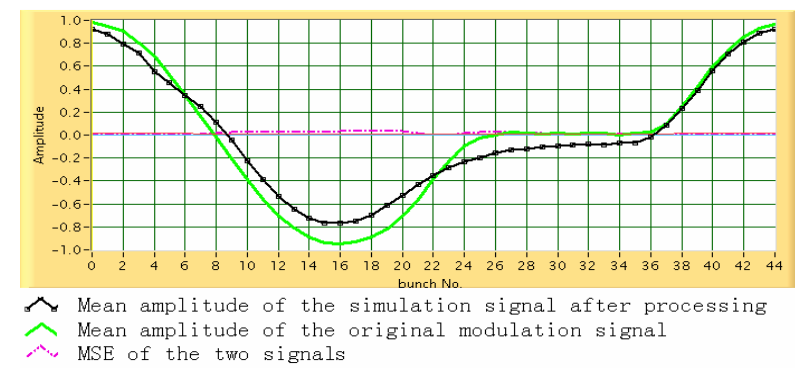

Figure 5: Simulation beam signal detected by ADC.

The test of the $\mathrm{BxB}$ measurement system by the simulation signal shows a good result of this high speed $\mathrm{AD}$ system. It is necessary before the experiment in the storage ring.

\section{EXPERIMENTS AND ANALYSES}

Restricted by the on broad memory of the digitizer, we couldn't acquire more than 4,194,192 samples in each record. With an external trigger of Revolution Frequency, we can trace each bunch during a long period. The marker of the bunch was given in the first data record, and then we can track one or several bunch in the following records. In this way, the information of each bunch, such as $\mathrm{x}, \mathrm{y}$ and sum signal, could be saved during the injection, ramping, wiggler charging normal operation and wiggler discharging. The status of each record of the following experiments is shown in Table 1.

Table 1: Detail of the record.

\begin{tabular}{|l|l|}
\hline Record No. & Status \\
\hline $001 \sim 046$ & Injection \\
\hline $047 \sim 123$ & Ramping \\
\hline $124 \sim 160$ & $\begin{array}{l}\text { Decreasing FRF from 204.0554MHz to } \\
204.016 \mathrm{MHz}\end{array}$ \\
\hline $161 \sim 200$ & Correcting Closed Orbit \\
\hline $201-267$ & Wiggler Charging (WC) \\
\hline $268 \sim 305$ & Adding skew-quadrupole \\
\hline $306 \sim 400$ & Open for users \\
\hline
\end{tabular}

\section{Tune Test}

Tune shift in the both $\mathrm{X}$ and $\mathrm{Y}$ direction is measured in the HLS, the $y$-direction is shown in Fig. 6. Each point in the figure is calculated by 2048 samples of Bunch No.13 in each record by FFT (Fast Fourier Transform) Algorithm. During the injection, tune y is decreased; the peaks in No.12 and No.28 correspond to the beam lost during the injection.

During the process of wiggler charging, from record No. 201 to 267 , the effect of magnetic field causes the increase of tune $y$. There is a detectable beta oscillation in the y-direction without kicker just after adding skewquadrupole. Tune shift from record No. 306 to No. 400 is about 0.0014 .

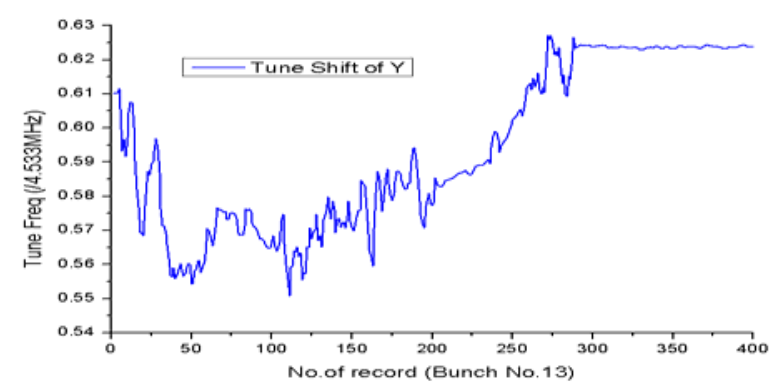

Figure 6: Tune shift in the Y-direction.

\section{Mode Test}

Mode test [3, 4] is also carried out by the data we acquired. All the samples of each record are used to undergo the FFT analyse. The corresponded amplitudes of the mode frequency are selected for the Fig. 7 and Fig.8. The phenomena of Fig. 7 and Fig. 8 are summed up in Table. 2 .

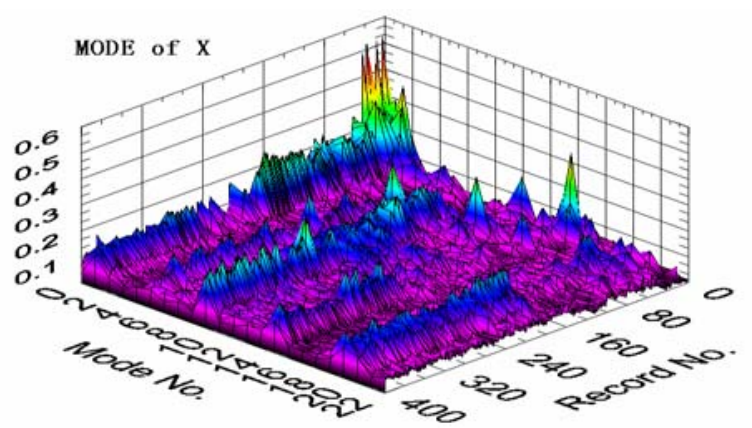

Figure 7: Mode display in X-direction.

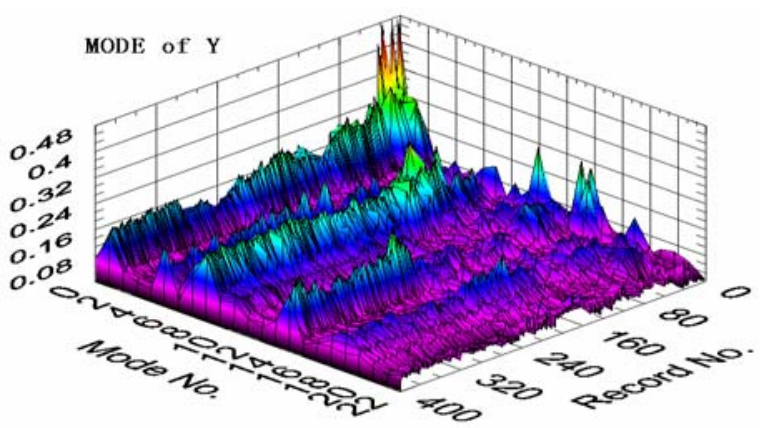

Figure 8: Mode display in Y-direction. 
Table 2: Analyse of the mode display.

\begin{tabular}{|l|l|l|}
\hline Mode No. & Phenomena & Direction \\
\hline 0 & Only high in injection & $\mathrm{X}$ and $\mathrm{Y}$ \\
\hline 1 & $\begin{array}{l}\text { Going down in adding } \\
\text { wiggler }\end{array}$ & $\mathrm{X}$ \\
\hline 5 & Going up in changing $\mathrm{F}_{\mathrm{RF}}$ & $\mathrm{X}$ \\
\hline $8(\mathrm{Y}) / 9(\mathrm{X})$ & Always high & $\mathrm{Y} / \mathrm{X}$ \\
\hline 14 & High in injection and WC & $\mathrm{X}$ and $\mathrm{Y}$ \\
\hline $\begin{array}{l}18(\mathrm{Y}) / 19 \\
(\mathrm{X})\end{array}$ & $\begin{array}{l}\text { Going up when correcting } \\
\text { the closed orbit }\end{array}$ & $\mathrm{Y} / \mathrm{X}$ \\
\hline
\end{tabular}

\section{Position and Amplitude Test}

Fig. 9 shows the average of the beam position in the Ydirection (see Eq. 2). The position changes a lot in the ramping and the decreasing of FRF.

$$
\bar{y}_{r, b}=\frac{1}{n} \sum_{i=1}^{n} y_{r, b, i}
$$

$\mathrm{n}=2048$ (in this case, Sampling length of each bunch)

$\mathrm{r}=1,2,3 \ldots 400$, (Record No.)

$\mathrm{b}=0,1,2 \ldots 44$, (Bunch No.)

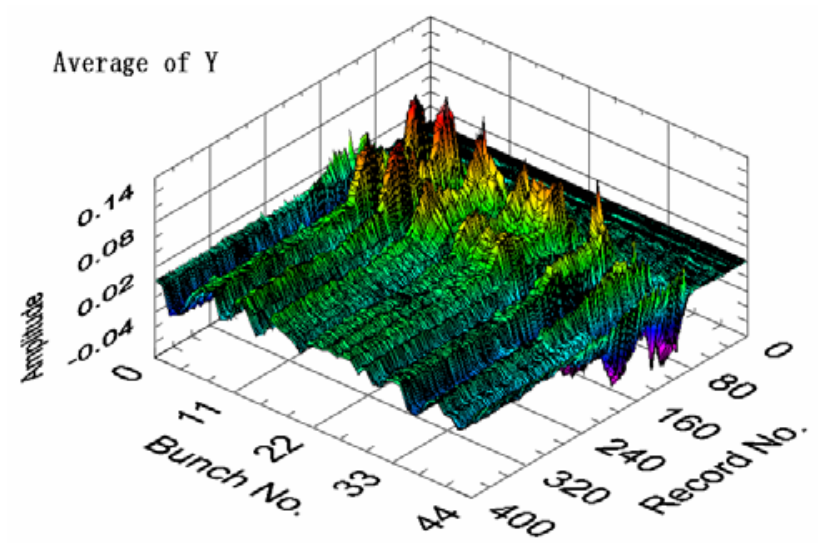

Figure 9: Average of beam position in the $\mathrm{Y}$-direction.

Fig. 10 shows the standard deviation of the beam position in the y direction (see Eq. 3); the measurement result shows a great change after the depressing FRF and correcting the closed orbit. The differences of the outcomes are caused by the transverse beta oscillation, the differences of the bunch current and the longitudinal oscillation. The bigger oscillation in the longitudinal may cause the bigger standard deviation in the transverse.

$$
S_{r, b}=\sqrt{\frac{1}{n} \sum_{i=1}^{n}\left(y_{r, b, i}-\bar{y}_{r, b}\right)^{2}}
$$

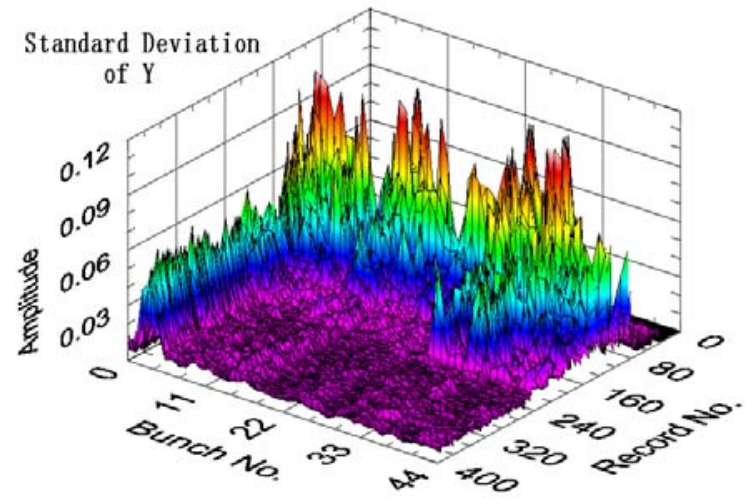

Figure 10: Standard Deviation of beam position in the Ydirection.

\section{FUTURE WORK}

After the succeed experiments of the BxB measurement system based on one BPM, the two BPMs measurement system and the $\mathrm{BxB}$ transverse feedback system will be established in the future. An on line phase space monitor of HLS based on this system is also under development.

\section{ACKNOWLEDGEMENTS}

The authors would like to present their thanks to Dr. Guangyao Feng and Prof. Hongliang Xu for their helpful discussion. Special thanks to Feng Zhao, who took in charge of the machine operation during the experiment.

\section{REFERENCES}

[1] W. H. Huang, H. S. Kang, D. T. Kim, J. Y. Huang and S. H. Nam, "Transverse Feedback System for PLS Storage Ring," Proc. of APAC, Beijing, China, 2001, pp 302-304.

[2] User Manual of 12 bits Digitizers by Acqiris.

[3] Mario Serio, "Multi bunch Instabilities and Cures," PAC, New York, 1999.

[4] Jianhong Liu, "The development of Bunch-by-Bunch measurement system and the research of instability in HLS," thesis for Doctor Degree of University of Science and Technology of China. 